\title{
Groupitizing reflects conceptual developments in math cognition and inequities in math achievement from childhood through adolescence
}

\author{
Mathieu Guillaume ${ }^{1}$, Ethan Roy ${ }^{1}$, Amandine Van Rinsveld ${ }^{1}$, Gillian S. Starkey ${ }^{2}$, Project iLead \\ Consortium, Melina R. Uncapher ${ }^{3}$, and Bruce D. McCandliss ${ }^{1}$ \\ ${ }^{1}$ Graduate School of Education, Stanford University, Stanford, CA \\ ${ }^{2}$ Center for Psychology, Goucher College, Baltimore, MD, USA \\ ${ }^{3}$ Department of Neurology, Weill Institute for Neurosciences, University of California, San Francisco, CA, USA
}

\begin{abstract}
Understanding the cognitive processes central to mathematical development is crucial to understanding disparities in math achievement. We investigated the "Groupitizing" ability in 1,209 U.S. third to eighth graders from 2016 to 2018 (mean age at first timepoint $=10.48,586$ girls, $39.16 \%$ Asian, $28.88 \%$ Hispanic/Latino, $18.51 \%$ White), a process capturing the ability to use grouping cues to access the value of a set. Groupitizing improves from late childhood to early adolescence $(d=3.29)$, is a central predictor of math achievement (beta weight $=.30)$, is linked to conceptual processes in mathematics (minimum $\mathrm{d}=0.69$ ), and explains the relation between socioeconomic status and mathematics achievement (minimum beta weight $=.11$ ) in ways that explicit symbolic measures may miss.
\end{abstract}

Keywords: Math achievement, Math ability, Number Sense, Enumeration, SES

Late childhood to early adolescence represents a crucial period of mathematical development in which conceptual structures are refined and automated (Artemenko et al., 2018; Whitaker et al., 2018), yet also a period of vulnerability in which achievement gaps widen significantly (Anderman, 1998), often creating entrenched patterns of inequity in math achievement that potentially persist over the life span. Better understanding how fundamental conceptual aspects of number cognition change over this developmental period is crucial to understanding how such abilities contribute to both development and inequities of broader math achievement. In the current study, we investigate how key aspects of mathematical development reflected in the "groupitizing" ability develop and help explain the relation between socioeconomic status and gaps in mathematics achievement in ways that usual explicit numerical and arithmetic assessments may miss.

\section{Subitizing and Groupitizing}

Humans, as well as non-human primates, have a singular ability to directly grasp the exact number of items in small sets up to four with remarkable accuracy and precision (Bourdon, 1908; Jevons, 1871; Nieder \& Miller, 2004). Kaufman et al. (1949) coined the term subitizing to characterize this ability, capturing the essence of sudden

Corresponding author: Bruce D. McCandliss
brucemc@stanford.edu

(subito) access to exact cardinal values. Subitizing allows for instant and accurate processing of small cardinalities, which substantially differs from serial counting (precise but time-consuming) and approximating (fast but imprecise) processes required to enumerate sets with more than four objects (Dehaene \& Cohen, 1994; Hyde, 2011; Hyde \& Spelke, 2009; Vuokko et al., 2013). For the latter processes, the greater the number of elements in the collection, the slower the serial counting, and the more imprecise the approximations; this phenomenon is commonly called the set size effect (Trick \& Pylyshyn, 1994).

Importantly, although subitizing relies on general attentional and object-tracking processes (Burr et al., 2010), this cognitive process is considered as one of the core systems of numbers since it allows the precise representations of distinct objects (Feigenson et al., 2004). Subitizing also plays a critical role in the development of numerical skills, mostly in helping young children grasp the meaning of the first number words (Benoit et al., 2004; Clements \& Sarama, 2011a). As such, subitizing was generally found to correlate with math achievement (Gray \& Reeve, 2014; LeFevre et al., 2010), but recent findings suggest that it does not predict math abilities (Anobile et al., 2019).

In 2010, McCandliss and colleagues (McCandliss et al., 2010) introduced a new construct to the lexicon of enumeration processes - groupitizing - to describe how exact enumeration speed is enhanced by grouping cues that segment a large set into subitizable subgroups of up to four items (following insights from Freeman, 1910). According to the authors, groupitizing rests on the conceptual understanding 
of cardinal values as composed of subsets: for instance, the value " 3 " is composed of three units (i.e., $3=1+1+1$ ) but " 3 " can also be composed of one subset of two units and another subset of one unit (i.e., $3=2+1$ ). In a subsequent study, G. S. Starkey and McCandliss (2014) further showed that groupitizing emerged after kindergarten and continued to grow through 3rd grade; and that fluent groupitizing (primarily within the oldest age groups) reduced the set size effect on enumeration speed. Furthermore, groupitizing was linked to individual differences in symbolic math fluency. In fact, groupitizing accounted for unique variance in symbolic math fluency above and beyond the influence of subitizing, counting fluency, and Approximate Number System (ANS) acuity (see Feigenson et al., 2004).

The groupitizing paradigm introduced by McCandliss et al. (2010) included several key elements which may be uniquely suited to capturing key aspects of development of number cognition relevant to understanding math achievement. The task is fluency-based using low-difficulty trials that even very young children can handle with high accuracy and minimal instructions (i.e.,"How many dots all together?"). The central construct is evident as an effect caused by a minimal experimental manipulation across two conditions, the presence or absence of grouping cues, which has advantages of isolating cognitive processes of interest. The stimuli are entirely novel and non-symbolic, each consisting of a set of up to 9 dots, avoiding symbolic or welllearned canonical stimulus patterns (e.g., dice patterns, see Mandler and Shebo, 1982), thus diminishing the influence of retrieval processes and memory access that might be associated with well-practiced symbolic problems encountered frequently in school, and instead emphasizing how children generalize their number knowledge to solve novel problems. Finally, the assessment of the key groupitizing processes is entirely implicit. Children are given minimal instructions to indicate how many dots they see and encounter mixed blocks of dot arrays that are unstructured or contain grouping cues. As such, this paradigm may be sensitive to children's spontaneous orientation toward number processes, which can often account for unique variance in achievement that are missed by more explicit assessments (Hannula \& Lehtinen, 2005; Hannula et al., 2010).

In the years since McCandliss' study of groupitizing in children, extensive research in adults have uncovered two key processes involved in groupiziting - the first linked to access to cardinal values of subsets, and the second linked to more conceptual mathematical processes involved in combining cardinal values of subsets into exact cardinal values of entire sets. In particular, recent studies showed that groupitizing involves subitizing processes at an early stage (Anobile et al., 2020; Maldonado Moscoso et al., 2020; Wege et al., 2021). Note that, fascinatingly, groupitizing does not limit to visual scenes but can be found in auditory sequences too, through subitizations of grouped auditory targets (Anobile et al., 2021). Grasping the cardinality of each subgroup through subitization is thus the first key numerical process required in groupitizing. A recent study showed that, after the subitization step, the subitized values are used as operands in the mental combination operated to get the total number of items (Ciccione \& Dehaene, 2020). The authors reported that combining dissimilar cardinal values (such as three subgroups of 3,2 , and 1 items) afford mental additions of these values (e.g., $3+2+1)$ to get the total. The ability to use mental arithmetic to get the correct response - in the absence of math symbols - is the second key conceptual process of groupitizing. It should be noted that these key processes are derived from data collected in adults; while the subitization processes of the first stage should be stable from age six onward (P. Starkey \& Cooper, 1995), the mental computation processes involved in the second stage are likely to change over the course of development. Thus, additional data on young people are still needed to understand how groupitizing develops.

\section{The current study}

We examine the role of groupitizing for the conceptual and procedural development of mathematics that unfolds over the subsequent years leading up to high-school entry, by partnering with a large semi-urban school district to collect data on a diverse group of learners using a novel tablet-based assessment (see Younger et al., 2021), with three specific objectives.

First, we set out to explore, for the first time, how groupitizing benefits continue to develop beyond early elementary school, and how such developments relate to emerging mathematical abilities and educational achievement. This motivated us to engage the largest, most demographically diverse sample $(N=1,209)$ of children assessed in groupitizing to date. Furthermore, to better link groupitizing development to educationally relevant achievements in math, in addition to cognitive assessments of arithmetic fluency that closely paralleled our previous developmental groupitizing study (G. S. Starkey \& McCandliss, 2014), we also examined how groupitizing related to grade-level appropriate developments in mathematics as assessed by educational institutions. We used the state standardized test scores for Mathematics (Smarter Balanced Assessment System for California, SBAC) as the central outcome measure in a multivariate regression approach, with a central aim to differentiate specific contributions of groupiziting ability over and above mathdomain-specific abilities such as math fluency or more general abilities outside the domain of math such as executive functions or reading comprehension.

Secondly, we set out to better understand the cognitive and conceptual processes involved in the development of groupitizing. We thus introduced experimental manipulations de- 
signed to impact within subgroup processes (i.e., the processing of each cardinal value) and between subgroup processes (i.e., the combination of the subitized values, see Figure 1a). We investigated the subgroup composition by first manipulating the maximal value in subgroups. One composition condition ("submax 4") maximized the dissimilarity of subgroup values, thus encouraging strategies of anchoring computations on the maximal value and adding smaller values - while still remaining in the subitizing range for most children. For example, for the value of 7 , we presented a maximal subgroup of 4 , paired with a subgroup of 2 and 1 (yielding the addition $4+2+1$ ). As children come to master arithmetic strategies such as finding the maximum value and adding smaller values (Butterworth et al., 2001; Groen \& Parkman, 1972), fluency in conceptual processes of combining subgroups should be enhanced here. Alternatively, we created another composition to maximize similarity of subgroups ("submax 3"), as in the case of seven being composed of 3,2 , and 2 (i.e., $3+2+2$ ), which seeks to maximize the similarity of the subgroup values to minimize such strategies. Under the view that groupitizing development beyond the middle of elementary school is dominated by the emergence and refinement of children's abilities to apply conceptual processes in mathematics to novel stimuli (following Clements and Sarama, 2011a, 2011b), neither subgroup composition condition should demonstrate an advantage early on, but as conceptual expertise emerges, an advantage for "submax 4" condition should emerge. Conversely, under the view that groupitizing is dominated by increasing abilities to access the cardinal values from subgroups, quite a different developmental pattern would result. Children would consistently demonstrate faster access to small sets of 3 relative to small sets of 4 , driven largely by the fact that although many children have a subitizing range that includes 4 , this ability develops slowly over development (Paliwal \& Baroody, 2020). Thus we might see an early advantage for "submax 3 " over "submax 4" for the youngest participants, and this advantage would tend to shrink over time as more children develop increasing subitizing spans that encompass four objects.

To manipulate how subgroups are combined, we asked how groupitizing is impacted when grouped arrays of the same value were formed by 2 versus 3 subgroups. Subitizing processes have been shown to occur in parallel across twoand even three-group displays (Wege et al., 2021), which opens up the possibility of direct access to the cardinality of an entire set, with similar latencies for two and three subgroup sets. However, recent findings suggest that groupitizing demonstrates several remarkable parallel effects that also impact symbolic arithmetic (Ciccione \& Dehaene, 2020) including a large effect caused by the number of operands (e.g., two versus three operand addition). This number of operands effect potentially reflects an iterative mental addition process such that an additional subgroup would require an addition mental step. For instance, one subgroup of 3 dots and one subgroup of 2 dots would yield the addition $3+2$, and one subgroup of 3 dots, and two subgroups of 2 dots would yield the addition $3+2+2$. As such, contrasting groupitizing performance for two versus three subgroup collections may shed light on the development of such iterative conceptual processes central to combining groups.

Third, and finally, we embarked on a novel exploration of how groupitizing might serve to mediate the well-established relation between the Socioeconomic Status (SES) and math achievement (e.g., Arnold and Doctoroff, 2003; Caro, 2009; Duncan et al., 2010; Morrissey et al., 2013). SES is an aggregated sociological index of multiple factors, many of which reflect systemic inequities in access to appropriate educational opportunities, which in turn are reflected in metrics of development of language (Fernald et al., 2013) and math skills (Demir-Lira et al., 2016). Regarding school (and math) achievement, it is known that teacher effectiveness is more uneven in low-SES school (Nye et al., 2004). We speculate that children in environments associated with lower SES indicators may have less access to educational resources (e.g., skilled teachers) that facilitate development of robust flexible number representations, such as conceptual processes of grouping that underlie arithmetic skills. Inequalities in mastering the groupitizing concepts of combining and grouping numbers could then be related to differences in mathematics achievement. As groupitizing provides a novel, nonsymbolic assessment of the conceptual structure of mental arithmetic (Ciccione \& Dehaene, 2020) such as fluency and flexibility in accessing knowledge of how groups of numbers combine, it may prove useful in better understanding the relation between SES and math achievement. To the extent that this relation is mediated by groupitizing, it might suggest that conceptual and combinatorial representations of number make up an important aspect of the well-established link between SES measures and math achievement, which can be assessed via this implicit measure via non-symbolic stimuli.

We hypothesize that groupitizing performance largely reflects foundational conceptual aspects of mathematical development spanning elementary through high school entry, and thus predict that individual variations in non-symbolic groupitizing will emerge as a central predictor of academic achievement in math, as assessed by a broad range of gradeappropriate math challenges in high-stakes state-mandated tests. Furthermore, as an assessment of such conceptual processes, we predict that groupitizing should be highly related to tests of symbolic math fluency, yet as a non-symbolic assay using untrained novel stimuli, that groupitizing should capture unique aspects of variation in mathematics achievement which may provide additional insights into the nature of SES based achievement gaps. 


\section{Method}

\section{Participants}

The current study is an exploratory analysis that is part of a mixed-longitudinal assessment involving typically developing children from nine schools (seven public, one private, one parochial) from northern California (Younger et al., 2021). Three different cohorts of children participated in a longitudinal assessment over two years from Fall 2016 to Spring 2018 (3rd-4th, 5th-6th, and 7th-8th graders). We administrated the dot enumeration task once per grade to 1,208 unique children in our sample (mean age at first timepoint: 10.48 years, 586 girls, 39.16\% Asian, 28.88\% Hispanic/Latino, $18.51 \%$ White, $13.45 \%$ Other or Multiple). We used the MAD procedure (Leys et al., 2013) and excluded seven children who performed beyond three standard deviations from the median performance (regarding both accuracy and correct latency) in the experimental task. The final sample consisted of 301 unique children in the first cohort, 384 children in the second cohort, and 516 children in the third cohort. In the current study, we did not analyze the longitudinal aspect and considered the following sample: 228 children (115 girls, mean age $=8.1$ years $)$ in 3rd grade, 271 children (132 girls, mean age $=9.5$ years $)$ in 4 th grade, 280 children (112 girls, mean age $=10.0$ years) in 5 th grade, 295 children (135 girls, mean age $=11.3$ years $)$ in 6th grade, 501 children ( 247 girls, mean age $=11.9$ years $)$ in 7 th grade, and 420 children ( 210 girls, mean age $=13.1$ years $)$ in 8 th grade .

We performed the study following protocols approved by the Institutional Review Board (IRB) of the University of California San Francisco. We obtained written parental (or guardian) consent from all participating children at the beginning of the study and verbal assent from all participating children before data collection. We obtained parental (or guardian) consent to receive state standardized test scores (Smarter Balanced Assessment System for California, SBAC) for Mathematics over the two assessment years (Year 0 and Year +1 ). We also obtained consent to receive information regarding both parents education level and the yearly parental income. Non-participating children received curricular instruction or worked on other schoolwork during the experiment. At the end of the study, all children (regardless of participation) received snacks and stickers.

\section{Dot enumeration task}

\section{Material and procedure}

Stimuli consisted of black dots on a gray background. We constructed the stimulus sets in a similar manner to those used in (G. S. Starkey \& McCandliss, 2014). Dots were either 3 or $6 \mathrm{~mm}$ in diameter (uniform size within an array), and arrays spanned a field area of either 5 or $10 \mathrm{~cm}$ in diameter. We constructed our experimental arrays with six, seven, or eight dots, and manipulated the distance between the dots: in Ungrouped arrays, we maintained the smallest distance between any pair of dots constant; in Grouped arrays, we maintained the smallest distance between the pairs of dots within each subgroup constant, and we made the smallest distance between any pair of subgroups at least three times the distance within each subgroup, see Figure 1a for illustrations. Within grouped arrays, we manipulated the number of subgroups ( 2 vs. 3 subgroups) and the maximal value in subgroups ( 3 vs. 4 dots, see Paliwal and Baroody, 2020). In addition, we used arrays with one to five dots and arrays with nine dots as fillers in the enumeration task to ensure equal probability of the responses.

Every child took part in the enumeration task on an individual iPad. The inter-stimulus interval was $1 \mathrm{~s}$. There were 60 experimental and 28 filler trials during the task. Two filler items were additionally administered as practice trials at the beginning of the task to ensure child understanding, for a total of 90 trials. In further analyses, we excluded trials where response times were smaller than 0.2 seconds (s) or larger than $20 \mathrm{~s}(0.51 \%$ of all trials). Overall, the median duration of the enumeration task was 3 minutes and 47 seconds.

\section{Correct responses per minute}

In our analyses, we considered a composite measure that yields an integrated effect size accounting for a larger portion of the variance than Error Rate (ER) and Responses Times (RT): the Correct Responses Per Minute (CRPM). This measure is based on the Rate Correct Score (RCS, Woltz and Was, 2006, see Vandierendonck, 2017), the number of correct responses per unit of time (i.e., per minute). It is defined by the following expression: $C R P M_{T}=\frac{c_{T}}{\sum R T_{T}}$, where $c$ is the number of correct responses for the set of trials $T$ (or condition $T$ ) and the denominator is the sum of responses times for the set of trials $T$. For instance, a score of $50 \mathrm{crpm}$ in a condition means that the child solved on average 50 trials from the condition under consideration in one minute. Greater CRPM is positively associated with better performance. We report ER and RT performance in the Appendices.

\section{Math achievement}

To measure math achievement, we used the Smarter Balanced Assessment System for California (SBAC) for Mathematics. This test comes from a comprehensive suite of standards-aligned tools and resources that provide differentiated lessons and activities that can help teachers support student learning but are also designed to provide accurate measures of student achievement and growth (see https: //smarterbalanced.org/). The SBAC math test is tailored to each grade level and includes a variety of topics such as algebraic thinking, geometry, understanding fractions, problem solving, and reasoning in the lower grades (3rd to 5th grade), comparing ratios and proportional relations, expression and 
equations, statistics and probability, and function analyses in the upper grades (6th to 8th grade). The SBAC tests were assessed during the Spring semester both years (Year 0 and Year +1$)$.

\section{Domain-general cognitive tests}

We assessed domain-general cognitive abilities thanks to the Adaptive Cognitive Evaluation (ACE, see Younger et al., 2021) running on iPads. In this computerized adaptive battery test, the maximum response time limit for each trial changed across the task to match the response window based on the child's performance. Children received positive feedback (in green) after each correct response, negative feedback (in red) after each mistake, and "too slow" feedback (in yellow) after any late response. The feedback duration was 200ms, and the inter-stimulus interval was 1 second. Each test module provided a RCS as performance index. In the current study, we focused on the following modules: Basic Response Time, Stroop, Flanker, and Visual Span.

We first used a computerized Basic Response Time task to evaluate general processing speed. In this task, a target was displayed at the center of the screen; children were instructed to press a button as fast as possible after the onset of the target. Children had to use their left index finger for 20 trials, and their right index finger for the remaining 20 trials. This task started with a maximum response time limit of 500ms.

We then used a computerized version of the color-word Stroop task to evaluate inhibition (Mead et al., 2002). Children viewed colored letters that spelled a color and were instructed to identify the color of the text while ignoring the color spelled by the letters. There were four possibilities: Red, Green, Yellow, or White. To respond, children were told to press the corresponding colored circle on the screen. We trained the response mapping during twenty-five practice trials, where they had first to match a central colored circle to the correct response (15 trials) and then practice the Stroop task (10 trials). After practice, children had to respond to 50 experimental trials (35 congruent and 15 incongruent trials). The task started with a maximal response time limit of $800 \mathrm{~ms}$.

We next used a computerized version of the letter Flanker task (Eriksen \& Eriksen, 1974) to evaluate selective attention. Children viewed an array of five letters displayed horizontally at the center of the screen. This array was composed of a combination of the following letters: $A, B, C$, or $D$. Children were instructed to identify the central letter while ignoring the flanking letters. To respond, children were told to press the location of the correct response at the bottom of the screen $(A, B, C$, or $D$, from left to right). There were 20 practice trials where only one letter was displayed at the center of the screen to learn the response mapping. After that, there were 50 experimental trials, with an initial maximum response time limit of $800 \mathrm{~ms}$.
We finally used a computerized version of the Corsi Block task (Corsi, 1973) to evaluate visuospatial working memory capacity. Children viewed a test grid of twenty black circles that were cued sequentially (lit in green), one at a time. In the Forward condition, children were instructed to repeat the previously seen sequence by directly tapping the correct circles, in the cued order. The experimental task was designed with levels and started with trials only containing three-location sequences. Once a child completed two consecutive trials of a level without error, they would advance to the next level that included an additional cued circle, increasing the difficulty level. Children completed as many levels as possible until they committed two consecutive errors, at which point the task ended. There was no time limit for this task. There were up to four practice trials with threelocation sequences. After the Forward condition, there was a Backward condition where children were instructed to tap the cued circles (lit here in blue) in the reverse sequence. To index performance, we added the values of the highest level reached in both conditions.

\section{Domain-specific cognitive tests}

We evaluated reading comprehension by adapting the Test of Silent Reading Efficiency and Comprehension (TOSREC, Wagner et al., 2010) on iPads. Children were instructed to read and determine whether a given sentence was true or false. Each sentence was displayed on the screen until a response was provided. Children were asked to correctly respond to as many sentences as possible in three minutes. To ensure that comprehension impacted scoring, we determined the reading comprehension score by subtracting the number of incorrect responses from the number of correct responses.

We finally assessed math fluency by using a timed assessment of single-digit arithmetic on iPads, based on commonly used metrics of math fluency (e.g., the Woodcock-Johnson III Tests of Achievement, Woodcock et al., 2007). Children were instructed to solve as many addition, subtraction, and multiplication problems as possible in three minutes. They were asked to respond by directly typing the correct answer. Each problem was displayed on-screen until the child responded. The operation difficulty progressively increased during the task. We determined the math fluency score as the total number of correct responses.

\section{Results}

\section{Measurement and statistical assessment}

In the main analyses, we considered a composite measure that yields an integrated effect size accounting for a larger portion of the variance than error rates and response time, the Correct Response Per Minute (CRPM, see Method). For each analysis, we built a full linear mixed effects model predicting CRPM with all relevant predictors as fixed or random factors. 
We then compared this full model to several reduced models, each missing a given predictor, using chi-squares tests on the log-likelihood values to determine whether the predictor under consideration was significant. These model comparisons were then used to assess the statistical significance of our experimental manipulations. We then estimated the effect sizes (Cohen's $d$ ) with the R 'esc' package (Lüdecke, 2017). We specify the full model used for each analysis in the following sections.

\section{Groupitizing effect and development}

We describe overall accuracy and latency in the appendices. Using the CRPM metric, we observed that children on average solved $18.25 \mathrm{crpm}, 95 \%$ Confidence Intervals (CI) $[17.95,18.54]$. To assess statistical significance, we built a full linear mixed effects model predicting CRPM, with grade (from 3rd to 8th), grouping condition (grouped or ungrouped arrays), set size ( 6,7 , or 8 dots) and their interactions as fixed predictors, and with participants and trials as random factors.

We observed the commonly found set size effect (Kaufman et al., 1949; Trick \& Pylyshyn, 1994) since children enumerated $19.42 \mathrm{crpm}$ for six-element arrays, 95\% CI [19.11, 19.72], $18.60 \mathrm{crpm}$ for seven-element, 95\% CI [18.29, 18.92], but only $16.96 \mathrm{crpm}$ for eight-element arrays, 95\% CI $[16.66,17.26]$. The size effect was significant, $\chi^{2}(2)=540.73, p<.001, d=1.79$. We also observed a significant effect of grade, $\chi^{2}(3)=855.78, p<.001, d=3.11$, illustrating children increasing performance across grades: 3rd graders solved $13.06 \mathrm{crpm}$, 95\% CI [12.44, 13.69], 4th graders solved $16.02 \mathrm{crpm}, 95 \%$ CI $[15.48,16.56], 5$ th graders solved 17.06 items crpm, 95\% CI [16.41, 17.71], 6th graders solved $19.00 \mathrm{crpm}, 95 \%$ CI [18.50, 19.51], 7th graders solved $19.95 \mathrm{crpm}, 95 \%$ CI [19.52, 20.37], and 8th graders solved $21.50 \mathrm{crpm}, 95 \%$ CI [20.99, 22.01].

We extended G. S. Starkey and McCandliss (2014)'s finding that the array structure impacted performance: children overall got $18.76 \mathrm{crpm}$ in grouped arrays, 95\% CI [18.45, 19.07], but only $16.97 \mathrm{crpm}$ in ungrouped arrays, 95\% CI $[16.69,17.24]$. The grouping condition effect was significant, $\chi^{2}(1)=883, p<.001, d=3.29$. Interestingly, we found that the size effect was reduced for grouped arrays relative to ungrouped arrays (see Figure 1b). The interaction between set size and condition was indeed significant, $\chi^{2}(1)=882.38, p<.001, d=3.28$, supporting the idea that performance was less impaired by the increasing set size in the Grouped condition. This is in line with G. S. Starkey and McCandliss (2014)'s observation that the set size effect decreases when children are enumerating grouped arrays.

We also found a significant interaction between grade and condition, $\chi^{2}(5)=34.687, p<.001, d=0.34$. As illustrated in Figure 1c, groupitizing - as indexed by improved enumeration for grouped relative to ungrouped arrays - continues to develop from 3rd through 8 th grade, extending G. S.
Starkey and McCandliss (2014)'s finding of continued grade by grade development of groupitizing skill from kindergarten to 3rd grade. This extended development was characterized by a significant groupitizing benefit present in 3rd grade that grew progressively stronger through 8th grade. We conducted pairwise Holm-Bonferroni-corrected t-tests (Holm, 1979) to compare grouped and ungrouped conditions within every grade. These analyses revealed that each grade level independently replicate the significant benefits for grouped over ungrouped arrays (3rd graders, $z=4.282$; 4th graders, $z=6.538 ; 5$ th graders, $z=7.970$; 6th graders, $z=10.412$; and 7 th graders, $z=10.489,8$ th graders, $z=10.678$; all ps $<.001)$.

\section{Groupitizing and math achievement}

\section{Performance in low vs. high math achievers}

One of the objectives of this study was to assess how groupitizing development was related to the development of math ability and more broadly to emerging math achievement. We assessed whether children who are more proficient in math differed in the enumeration task compared to the less proficient students. We categorized all children as a function of state standardized test scores for Mathematics (SBAC) obtained during the current school year. Within each grade, we differentiated the lowest (below the $25 \%$ percentile), the low (between the $25.1 \%$ and $49.9 \%$ percentiles), the high (between the $50 \%$ and $74.9 \%$ percentiles) and the highest achieving children (above the $75 \%$ percentile) in math. We observed that lowest math performers enumerated $14.43 \mathrm{crpm}, 95 \%$ CI [13.94, 14.92], low performers $17.42 \mathrm{crpm}, 95 \%$ CI [16.98, 17.85], high performers correctly $19.03 \mathrm{crpm}, 95 \%$ CI [18.53, 19.52], and the highest performers counted $23.03 \mathrm{crpm}, 95 \%$ CI [22.54, 23.52]. To assess statistical significance, we built a full linear mixed effects model with the child grade, the condition, and the math achievement group (and their interactions) as fixed factors and with participants and trials as random factors. Model comparison confirmed that enumeration performance was significantly affected by math achievement, $\chi^{2}(3)=459.35, p<.001, d=1.56$.

Interestingly, the analysis also revealed that the interaction between condition and math achievement was significant, $\chi^{2}(3)=239.04, p<.001, d=0.99$. Figure 1d clearly illustrates that the difference between enumerating grouped and ungrouped arrays increased with better math ability. In other words, a groupitizing advantage (i.e., the increase in performance related to the grouped structure) emerges across the different levels of math achievement. In particular, the lowest math achieving group showed no such an advantage. We note that as there was no instruction to use the array structure to solve the task, it is likely that the lowest achieving children simply enumerated all dots in a one-by-one fash- 


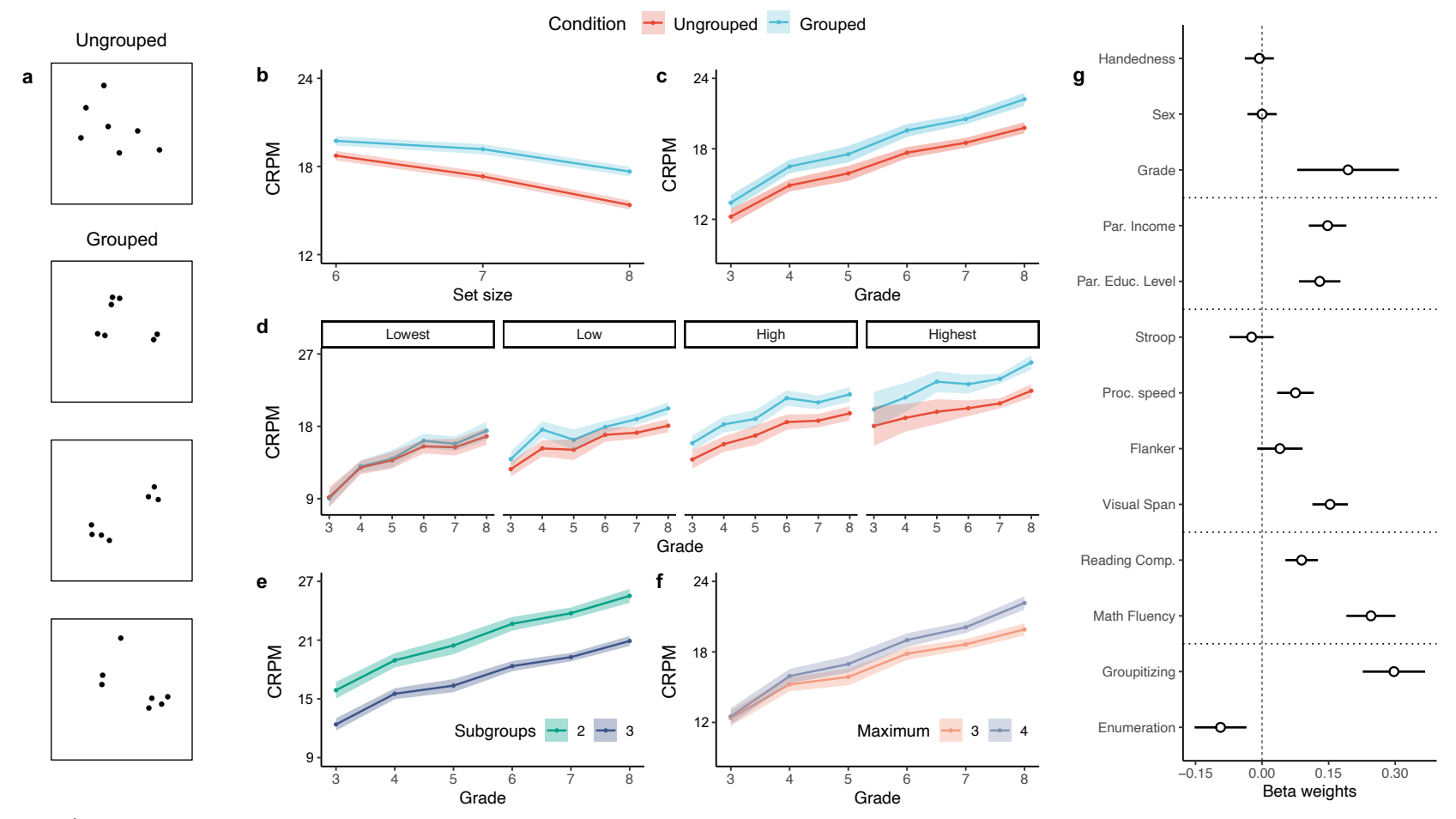

Figure 1

a. Illustration of the four stimulus conditions, when ungrouped, grouped into two and three subgroups, with a subgroup maximum of three or four; $\boldsymbol{b}$. Correct Response Per Minute (CRPM) as a function of condition and set size; $\boldsymbol{c}$. CRPM as a function of condition and grade; $\boldsymbol{d}$. CRPM as a function of math achievement (SBAC score, lowest: below the 25\% percentile, low: between the $25.1 \%$ and $49.9 \%$ percentiles, high: between the $50 \%$ and $74.9 \%$ percentiles, highest: above the $75 \%$ percentile), per grade; $\boldsymbol{e}$. CRPM for grouped arrays as a function of the number of subgroups, per grade; $\boldsymbol{f}$. CRPM for grouped arrays as a function of the maximal value in subgroups, per grade; $\boldsymbol{g}$. Beta weights of the best parsimonious regression model predicting math achievement. The colored areas and horizontal lines depict 95\% Confidence Intervals.

ion regardless of the structure of the structure of the arrays. This finding suggests that these children were either unable or uninclined to deliberately use the grouped structure to efficiently access the total of dots.

\section{Regression analyses}

To further investigate this observation, we assessed how groupitizing ability predicted math achievement via a full regression model designed to predict an math achievement scores with the following predictors: four individual/demographics predictors (Handedness, Grade, Race/Ethnicity, and Sex), two socioeconomic predictors (Parent Education Level, and Yearly Parental Income), four domain-general abilities predictors (Stroop, Flanker, Visual span, and Speed of processing), a domain-specific predictor for math (Math Fluency), a related educational achievement predictor (Reading Comprehension), and finally, our experimental manipulations: Enumeration (i.e., CRPM to ungrouped arrays) and Groupitizing (CRPM to grouped ar- rays). Handedness (1: 'Left-sided', 2: 'Right-sided'), Sex (1: 'Male', 2: 'Female'), Yearly Parental Income (1: 'Below $70 \%$ of state median', 2: 'Above $70 \%$ of state median'), and Parent Education Level (from 1: 'Did not graduate High School' to 5: 'Postgraduate level') were dummy coded as numeric variables. All numeric predictors and the dependent factor were standardized to obtain standardized beta coefficients (or Beta weights, see Figure 1g). The predictors in the full model, when combined, accounted for $60 \%$ of the variance in math achievement, $F(23,1448)=95.99, p<.001$, adjusted $R^{2}$ of .60. Critically, groupitizing accounted for the largest amount of this variance in math achievement (standardized beta weight $=.30,95 \%$ CI $[.26, .33])$.

To arrive at a parsimonious regression model that ranks the factors as a function of their contribution to the prediction, we used step-wise regression from the null model to the full model. The final model yielded a significant regression equation $F(17,1454)=128, p<.001$, with an adjusted $R^{2}$ of .60. Remarkably, among all general, demographic, 
socioeconomic, and individual factors, groupitizing was the strongest independent predictor of math achievement, with a Beta weight of .30, 95\% CI [.25, .35], and emerged as the first step of the model. Results from this parsimonious model replicated the findings from the full model analysis. Altogether, data support the notion that groupitizing is a critical predictor of math achievement.

\section{Effects of group composition}

The second objective was understanding the cognitive and conceptual processes involved in the development of groupitizing. We manipulated the number of subgroups ( 2 vs. 3 subgroups) and the maximal value in subgroups (submax 3 vs. 4) within grouped trials. We found out that the number of subgroups and the maximal value in each subgroup affected children's performance (see Figure 1e and 1f). Across all grades, children only got $17.60 \mathrm{crpm}$ for 3-subgroup arrays, $95 \%$ CI [17.31, 17.89], but they were able to get 21.64 crpm for 2-subgroup arrays, 95\% CI [21.25, 22.02]. Furthermore, they solved $17.07 \mathrm{crpm}$ with at most 3 dots in each subgroup, 95\% CI [16.77, 17.36], and they could solve 19.79 crpm arrays with a maximum of 4 dots in all subgroups, $95 \%$ CI [19.45, 20.13]. Interestingly, visual inspection of Figure $11 \mathrm{e}$ and $1 \mathrm{f}$ suggests that the advantage of enumerating two subgroups over enumerating three subgroups was constant across the grades, whereas the advantage of enumerating arrays with larger subgroups increased throughout grades.

To assess the significance of the results, we fitted a full linear mixed effects model predicting CRPM within grouped trials, with the child grade (from 3rd to 8th), the number of subgroups ( 2 vs. 3 ) and the maximal value in subgroups ( 3 vs. 4) and their interaction with the child grade as fixed predictors, and with participants and trials as random factors. This analysis revealed that there were significant effects of grade, $\chi^{2}(1)=561.73, p<.001$, the number of subgroups, $\chi^{2}(1)=1027.8, p<.001, d=4.76$, and the maximal value in a subgroup, $\chi^{2}(1)=131.05, p<.001, d=0.69$. Interestingly, the maximal value in a subgroup significantly interacted with the child grade, $\chi^{2}(1)=42.68, p<.001, d=0.38$, showing that the ability to benefit from grouped structure where the maximal value was above 3 improved with grade (and supposedly, age). Holm-Bonferroni-corrected t-tests revealed that 3 rd and 4th graders could not take advantage of grouped arrays with 4 dots, respectively $p=.530$ and $p=.053$, while older children could (5th graders, $p=.038$, 6th graders, $\mathrm{p}=.014,7$ th graders, $p<.001,8$ th graders, $p<.001)$. Lastly, the number of subgroups did not interact with the grade, $\chi^{2}(1)=0.210, p=.646, d=0.02$, supporting the idea that the number of subgroups had a constant cognitive cost in the enumeration procedure across grades.

\section{Groupitizing and socioeconomic status}

Performance in low vs. medium/high household income group The third objective was exploring whether and how groupitizing serves to mediate the well-established relation between the parental socioeconomic status (SES) and children's math achievement scores (e.g., Duncan et al., 2010; Morrissey et al., 2013). We previously highlighted how groupitizing ability also accounts for unique variance in math achievement, and thus we explored the role of parental income in understanding groupitizing performance. To do so, we used a binary income factor for the model, flagging children from households whose net annual earnings fell below $70 \%$ of the state median income (see Younger et al., 2021). We then built a full linear mixed effects model predicting CRPM with grade, grouping condition, array size, income, and their interactions as fixed predictors, and with participants and trials as random factors.

Inclusion of parental income significantly improved the model's performance for the enumeration task, $\chi^{2}(1)=$ $79.89, p<.001, d=0.53$. This means that children from low-income household had a significantly more difficult time in enumerating overall. Strikingly, a significant interaction between income and condition emerged, $\chi^{2}(1)=87.29, p<$ $.001, d=0.55$; children from households reporting lower income showed no groupitizing advantage (see Figure 2a). In contrast, children from households with incomes above this level demonstrated robust groupitizing advantages. The triple interaction between grade, condition, and income was significant, $\chi^{2}(16)=137.37, p<.001, d=0.71$. A series of Holm-Bonferroni-corrected t-tests revealed that children from an average or higher income household showed a groupitizing advantage within each grade (all $p s<.001$ ) but the picture was different from children from the low income group, where 3rd graders, $p=.084$ and 4th graders, $p=.251$, did not significantly groupitize. These findings emphasise that groupitizing indexes a cognitive ability that is associated with the many important differences captured by SES indicator variables, and that result cannot be readily reduced to differences in more general processes such as perception, processing speed, or even enumeration processes specific to mathematics.

\section{Mediation analyses}

Given the well-established association between SES and math achievement, and parallel findings linking groupitizing to math achievement, we conducted mediation analyses to assess whether a) groupitizing mediates the relation between income and math achievement and b) groupitizing mediates the relation between parent education level and math achievement.

The effect of yearly parental income on the state math achievement score (SBAC) was significantly mediated via 

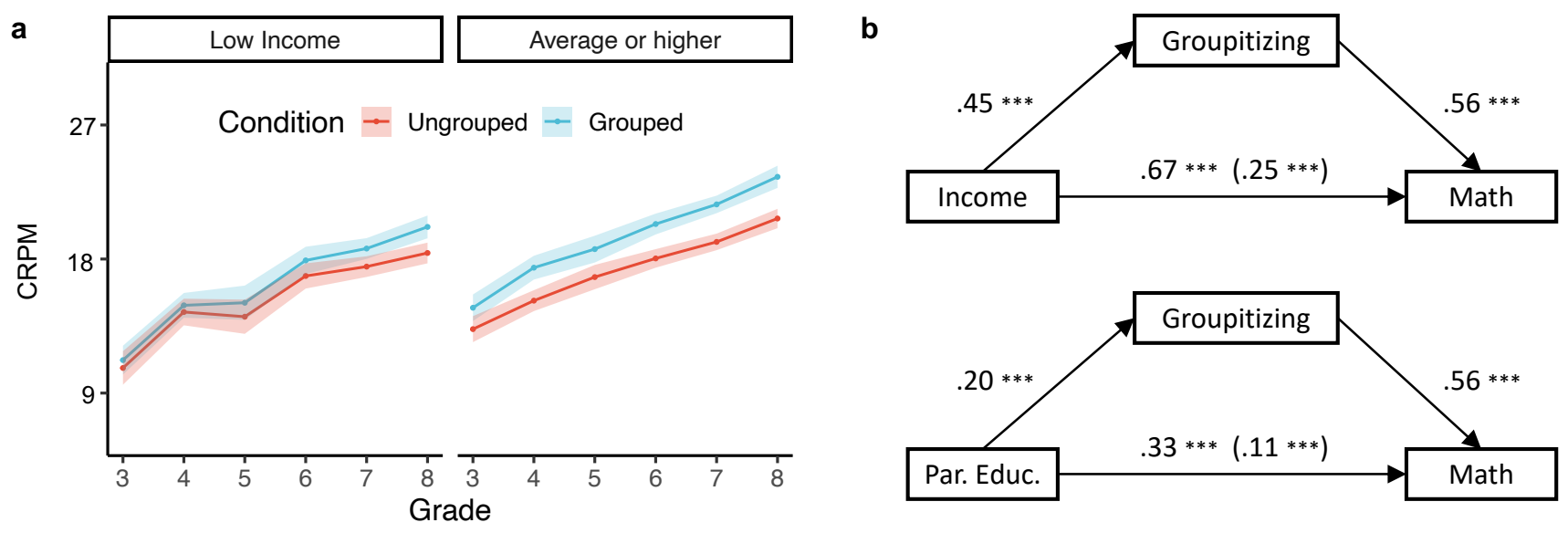

Figure 2

a. Correct Response Per Minute (CRPM) as a function of yearly parental income (low or average or higher income), per condition and grade. The colored areas depict $95 \%$ Confidence Intervals; $\boldsymbol{b}$. Beta coefficients for the relation between yearly parental income and math achievement (SBAC) and between parental education level and SBAC as mediated by the groupitizing ability. The coefficient between the predictor and math achievement, controlling for groupitizing, is in parentheses. *** $p<.001$

groupitizing ability. The standardized regression coefficient between income and math achievement and the regression coefficient between groupitizing and math achievement were significant, $p<.001$ (see top of Figure 2b). The indirect effect was $(.45)(.56)=.25$. We tested the significance of this indirect effect using bootstrapping procedures (1,000 samples). The bootstrapped indirect effect was $.252,95 \%$ CI [0.190, 0.322] and was significant, $p<.001$.

The effect of parental education level on the SBAC score was significantly mediated via groupitizing ability. The standardized regression coefficient between parent education level and math achievement and the regression coefficient between groupitizing and math achievement were significant, $p<.001$ (see bottom of Figure 2b). The indirect effect was $(.20)(.56)=.11$. We tested the significance of this indirect effect using bootstrapping procedures (1,000 samples). The bootstrapped indirect effect was .112, 95\% CI [0.083, 0.150] and was significant, $p<.001$.

\section{Discussion}

Overall, we replicated McCandliss et al. (2010)'s findings that the set size effect (Jevons, 1871; Kaufman et al., 1949; Mandler \& Shebo, 1982; Trick \& Pylyshyn, 1994) tends to disappear when arrays are grouped, meaning that children do not systematically enumerate all elements one-by-one but rather use grouping strategies to get to the total number (see also Anobile et al., 2020; Ciccione and Dehaene, 2020; Maldonado Moscoso et al., 2020; Wege et al., 2021). Once again, we emphasize that there were no explicit instructions towards grouping dots, so that the groupitizing phenomenon captures the adaptive ability to use the array properties for faster and more efficient processing. This provides evidence that most children can conceptually take advantage of the grouped structure of an array to get to its cardinality.

We further extend G. S. Starkey and McCandliss (2014)'s observation that the groupitizing advantage continues to increase from 3rd to 8th grade. We must acknowledge that we used a mixed longitudinal design with three different cohorts tested twice, which leads to two limitations to the study. First, data from different cohorts cannot directly describe continuous improvement from 3rd to 8th grade, and second, we cannot rule out the possibility that some practice effects led children to apprehend the task differently on their first and second attempts the following year. Nevertheless, our findings support that late childhood to early adolescence represents a crucial period of mathematical development in which conceptual structures are refined and automated (Artemenko et al., 2018; Whitaker et al., 2018).

\section{Groupitizing uniquely predicts math achievement}

Remarkably, youth with different math achievement levels showed distinctive patterns of groupitizing ability: while the highest performing children showed a groupitizing advantage across all grades, the lowest performing children did not show such an advantage at any grade. In other words, the lowest achieving 8th graders were not able to use the grouping properties to solve the task whereas the highest performers in 3rd grade could. This is a substantial delay of five years in a task that involves low-difficulty trials that even very young children can handle with high accuracy and 
minimal instructions. This delay most likely reflects conceptual gaps in the two keys processes involved during groupitizing, that is getting to the cardinal values of each subset, and then combining these values. Catching up on such conceptual gaps in this critical period of mathematical development might be very challenging for the lowest achieving youth (see Anderman, 1998), and should deserve future attention. We acknowledge that we cannot directly relate our selection criterion (i.e., the lowest group performing below the $25 \%$ percentile of the SBAC) to mathematical disabilities, but such findings illustrate that dyscalculic patients are likely to display a conceptual deficit in groupitizing. Future studies are however still needed to evaluate the extent to which children with specific mathematical disabilities struggle with groupitizing.

Regression analyses supported the existence of a tight relation between the groupitizing ability and math achievement at the whole sample level. We fitted models that accounted for socioeconomic, domain-general and domain-specific factors and we found that the groupitizing ability was the best cognitive predictor of math achievement. Our findings provide evidence that groupitizing not only correlates with math fluency (see zero-order correlations in Appendices, as also reported by Maldonado Moscoso et al., 2020; G. S. Starkey and McCandliss, 2014) but also predicts math achievement in a way that symbolic arithmetic measures may miss. Our study design, however, does not readily permit interpretation of direction of causality, and thus our claims about such issues should appropriately be restricted.

\section{Groupitizing reflects math concepts}

We analyzed the group composition to better characterise the groupitizing procedure from a cognitive viewpoint. We found that grouped arrays with 2 subgroups yielded better performance than arrays with 3 subgroups. There is thus a clear cognitive cost associated with groupitizing an additional subgroup. Interestingly, the cognitive cost was constant across the grades in our data set, suggesting that the cognitive processes involved in this cost did not develop through late childhood to early adolescence. One could assume that part of this cost is due to visuo-spatial scanning strategies to subsequently gaze towards each subgroup. However, a recent study suggested that multiple subitizing processes can occur in parallel (Wege et al., 2021), so this scanning component should have little effect on the cost. Another non-mutually exclusive interpretation likely explains most of the cognitive cost: combining three cardinal values takes more time than combining two values. Recent findings showed that groupitizing involves mental arithmetic (Ciccione \& Dehaene, 2020), such that combining an additional subgroup yield an additional step in the mental addition of the subtotals. In other words, adding an additional addend takes time; this processing time being stable in our data set, which suggests that groupitizing requires arithmetic conceptual manipulations that are mastered in 3rd grade.

We further found that children were better to groupitize 4 and 1 dots ("submax 4") than 3 and 2 dots ("submax 3"). Note that this observation nicely mirrors well-known arithmetic data showing that additions such as $4+1$ yield better performance than $3+2$ (Groen \& Parkman, 1972). Furthermore, this effect interacted with grade: 3 rd and 4 th graders did not initially show any clear advantage in groupitizing either subsets with 3 or 4 items, but a groupitizing advantage arose within older children who were able to use grouping cues on "submax 4" more and more efficiently in comparison to "submax 3" arrays. This result pattern is not in line with the idea that groupitizing development is dominated by increasing subitizing abilities (see Paliwal and Baroody, 2020. Alternatively, our results are consistent with the view that groupitizing development beyond the middle of elementary school is dominated by the emergence and refinement of children's abilities to apply conceptual processes in mathematics to novel stimuli (following Clements and Sarama, 2011a, 2011b).

From a more general cognitive perspective, we emphasise that groupitizing might also be viewed as an efficient strategy that inherently emerges from the context (following Siegler, 2007). Grouping is only possible when objects are sufficiently clustered together (within a window size of $4^{\circ}$ of visual angle, Im et al., 2016). The grouped structure of a given stimulus thus serves as a critical cue to elicit the groupitizing behavior. Subsequently, children who used groupitizing when possible needed to process the structure information and detect that (partial or complete) grouping is possible before starting the groupitizing procedure. In this context, groupitizing likely involves several cognitive control processes at the earliest stage, in addition to the attention-based mechanisms (Maldonado Moscoso et al., 2020) and later mental arithmetic processes (Ciccione \& Dehaene, 2020). That being said, results from our regression analyses show that the relation between groupitizing and math achievement cannot be reduced to relations between these executive functions, cognitive control, and attention-based processes with math achievement.

\section{Groupitizing mediates SES and math achievement}

The socioeconomic factors we considered in this study (parent education level and yearly parental income) significantly predicted math achievement. This observation replicates the common finding that SES tightly relates to math achievement (e.g., Caro, 2009; Duncan et al., 2010; Morrissey et al., 2013). We focused on the yearly parental income and found that children from the low income group did not show the same groupitizing advantage than the other group. Strikingly, low income children before 5th grade could not efficiently groupitize, whereas 3rd graders from an average 
income household (or higher) were able to do so. This result was quite similar to the groupitizing gap we found between children with low math scores and those with high math scores. A limitation of the current study is that we considered household income as a dichotomous factor (annual earnings below or above $70 \%$ of the state median income), and our criterion may not capture the subtle patterns that link SES and math achievement. Thus, more fine-grained studies are needed to corroborate and extend our findings.

We further looked at the relation between SES and math achievement, and found that groupitizing significantly mediates this relation. Our findings provide evidence that socioeconomic status predicts groupitizing behavior, which in turn predicts math achievement. These results illustrate that groupitizing requires cognitive abilities (i.e., grouping and combining cardinalities) that, on the one hand, are largely influenced by SES and, on the other hand, predict mathematics outcomes. We suggest that children of low SES may have less access to educational resources (i.e., teachers, see Nye et al., 2004), which impairs mastery of the mathematics concepts not only involved in groupitizing, but also related to differences in mathematics achievement. This is an important finding since we can implicitly measure - and supposedly train - the groupitizing ability in non-symbolic numerical tasks that involves low-difficulty trials. Future training studies could lay the groundwork for remediation studies aimed at improving the groupitizing ability within lowincome children in the hope of improving math skills and outcomes.

\section{Conclusion}

Our study emphasises that groupitizing is a cognitive ability that predicts math achievement over and above socioeconomic, domain-general and domain-specific factors. Groupitizing cannot be reduced to math fluency or more generally math ability, but it does involve mental arithmetic processes. This is likely the reason why it strongly correlates with math ability and uniquely predicts math achievement. Furthermore, we claim that groupitizing is an interesting proxy to assess non-symbolic arithmetic. As such, groupitizing seems to be an appropriate tool to evaluate and train mental arithmetic amongst some at-risk populations, such as low income children or dyscalculic patients. We believe in this context that groupitizing might be an interesting asset in the future to diagnose and remedy specific math disabilities. More generally, we advise future studies aiming at evaluating numerical abilities to measure groupitizing.

\section{Acknowledgments}

This research was supported by funding from the National Science Foundation, Science of Learning Collaborative Networks Grant (NSFSLCN-1540854) awarded to Melina Uncapher (lead Principal Investigator [PI]) and Adam Gazzaley, and co-PIs Joaquin Anguera, Silvia Bunge, Fumiko Hoeft, Bruce McCandliss, Jyoti Mishra, and Miriam RosenbergLee. The study was approved by the Institutional Review Board (IRB) of the University of California San Francisco. The authors are thankful to the students, parents, teachers, school district administrators, and all members of the consortium. The data, analytic code, and materials necessary to reproduce the analyses presented here are not publicly accessible. The analyses presented here were not preregistered.

\section{References}

Anderman, E. M. (1998). The middle school experience: Effects on the math and science achievement of adolescents with LD. Journal of Learning Disabilities, 31(2), 128-138. https : // doi . org/10 . 1177/ 002221949803100203

Anobile, G., Arrighi, R., \& Burr, D. C. (2019). Simultaneous and sequential subitizing are separate systems, and neither predicts math abilities. Journal of Experimental Child Psychology, 178, 86-103. https: //doi.org/10.1016/j.jecp.2018.09.017

Anobile, G., Castaldi, E., Maldonado Moscoso, P. A., Arrighi, R., \& Burr, D. (2021). Groupitizing improves estimation of numerosity of auditory sequences. Frontiers in Human Neuroscience, 15, 687321. https://doi.org/10.3389/fnhum.2021.687321

Anobile, G., Castaldi, E., Moscoso, P. A. M., Burr, D. C., \& Arrighi, R. (2020). "Groupitizing": A strategy for numerosity estimation. Scientific Reports, (1), 13436. https://doi.org/10.1038/s41598-020-681111

Arnold, D. H., \& Doctoroff, G. L. (2003). The early education of socioeconomically disadvantaged children. Annual Review of Psychology, 54(1), 517545. https://doi .org/10.1146/annurev. psych.54. 111301.145442

Artemenko, C., Soltanlou, M., Ehlis, A.-C., Nuerk, H.-C., \& Dresler, T. (2018). The neural correlates of mental arithmetic in adolescents: A longitudinal fNIRS study. Behavioral and Brain Functions, 14(5), 113. https://doi.org/10.1186/s12993-018-0137-8

Benoit, L., Lehalle, H., \& Jouen, F. (2004). Do young children acquire number words through subitizing or counting? Cognitive Development, 19(3), 291-307. https://doi.org/10.1016/j.cogdev.2004.03.005

Bourdon, B. (1908). Sur le temps nécessaire pour nommer les nombres. Revue Philosophique de la France et de l'Etranger, 65, 426-431.

Burr, D. C., Turi, M., \& Anobile, G. (2010). Subitizing but not estimation of numerosity requires attentional resources. Journal of Vision, 10(6), 20-20. https://doi. org/10.1167/10.6.20 
Butterworth, B., Zorzi, M., Girelli, L., \& Jonckheere, A. R. (2001). Storage and retrieval of addition facts: The role of number comparison. The Quarterly Journal of Experimental Psychology Section A, 54(4), 1005-1029. https://doi.org/10.1080/713756007

Caro, D. H. (2009). Socio-economic status and academic achievement trajectories from childhood to adolescence. Canadian Journal of Education, 32(3), 558590.

Ciccione, L., \& Dehaene, S. (2020). Grouping mechanisms in numerosity perception. Open Mind, 4, 102-118. https://doi.org/10.1162/opmi_a_00037

Clements, D. H., \& Sarama, J. (2011a). Early childhood mathematics intervention. Science, 333(6045), 968-970. https://doi.org/10.1126/science.1204537

Clements, D. H., \& Sarama, J. (2011b). Early childhood teacher education: The case of geometry. Journal of Mathematics Teacher Education, 14(2), 133-148. https://doi.org/10.1007/s10857-011-9173-0

Corsi, P. M. (1973). Human memory and the medial temporal region of the brain. Dissertation Abstracts International, 34(2-B), 891.

Dehaene, S., \& Cohen, L. (1994). Dissociable mechanisms of subitizing and counting: Neuropsychological evidence from simultanagnosic patients. Journal of Experimental Psychology: Human Perception and Performance, 20(5), 958-975. https://doi .org/10. 1037/0096-1523.20.5.958

Demir-Lira, Ö. E., Prado, J., \& Booth, J. R. (2016). Neural correlates of math gains vary depending on parental socioeconomic status (SES). Frontiers in Psychology, 7(892), 1-12. https://doi.org/10.3389/fpsyg. 2016.00892

Duncan, G. J., Ziol-Guest, K. M., \& Kalil, A. (2010). Earlychildhood poverty and adult attainment, behavior, and health. Child Development, 81(1), 306-325. https://doi.org/https://doi.org/10.1111/j.14678624.2009.01396.x

Eriksen, B. A., \& Eriksen, C. W. (1974). Effects of noise letters upon the identification of a target letter in a nonsearch task. Perception $\mathcal{F}$ Psychophysics, 16(1), 143-149. https://doi.org/10.3758/BF03203267

Feigenson, L., Dehaene, S., \& Spelke, E. (2004). Core systems of number. Trends in Cognitive Sciences, 8(7), 307-314. https://doi.org/10.1016/j.tics.2004.05.002

Fernald, A., Marchman, V. A., \& Weisleder, A. (2013). SES differences in language processing skill and vocabulary are evident at 18 months. Developmental Science, 16(2), 234-248. https://doi.org/https://doi. org/10.1111/desc. 12019

Freeman, F. N. (1910). Untersuchungen über den aufmerksamkeitsumfang und die zahlauffassung bei kindern und erwachsenen [studies on attentional scope and number perception in children and adults]. Pädagogisch-psychologische Arbeiten, 1, 88-168.

Gray, S. A., \& Reeve, R. A. (2014). Preschoolers' dot enumeration abilities are markers of their arithmetic competence. PLoS ONE, 9(4), e94428. https://doi. org/10.1371/journal.pone.0094428

Groen, G. J., \& Parkman, J. M. (1972). A chronometric analysis of simple addition. Psychological Review, 79(4), 329-343. https://doi.org/10.1037/h0032950

Hannula, M. M., \& Lehtinen, E. (2005). Spontaneous focusing on numerosity and mathematical skills of young children. Learning and Instruction, 15(3), 237-256. https://doi.org/10.1016/j.learninstruc.2005.04.005

Hannula, M. M., Lepola, J., \& Lehtinen, E. (2010). Spontaneous focusing on numerosity as a domain-specific predictor of arithmetical skills. Journal of Experimental Child Psychology, 107(4), 394-406. https: //doi.org/10.1016/j.jecp.2010.06.004

Holm, S. (1979). A simple sequentially rejective multiple test procedure. Scandinavian Journal of Statistics, 6(2), $65-70$.

Hyde, D. C. (2011). Two systems of non-symbolic numerical cognition. Frontiers in Human Neuroscience, 5(150), 1-8. https://doi.org/10.3389/fnhum.2011. 00150

Hyde, D. C., \& Spelke, E. S. (2009). All numbers are not equal: An electrophysiological investigation of small and large number representations. Journal of Cognitive Neuroscience, 21(6), 1039-1053. https : //doi.org/10.1162/jocn.2009.21090

Im, H. Y., Zhong, S.-H., \& Halberda, J. (2016). Grouping by proximity and the visual impression of approximate number in random dot arrays. Vision Research, 126, 291-307.

Jevons, W. S. (1871). The power of numerical discrimination. Nature, 3(67), 281-282. https://doi.org/10. 1038/003281a0

Kaufman, E. L., Lord, M. W., Reese, T. W., \& Volkmann, J. (1949). The discrimination of visual number. The American Journal of Psychology, 62(4), 498-525. https://doi.org/10.2307/1418556

LeFevre, J.-A., Fast, L., Smith-Chant, B. L., Skwarchuk, S.-L., Bisanz, J., Kamawar, D., \& Penner-Wilger, M. (2010). Pathways to mathematics: Longitudinal predictors of performance. Child Development, 81(6), 1753-1767.

Leys, C., Ley, C., Klein, O., Bernard, P., \& Licata, L. (2013). Detecting outliers: Do not use standard deviation around the mean, use absolute deviation around the median. Journal of Experimental Social Psychology, 49(4), 764-766. https:// doi .org/10.1016/j. jesp.2013.03.013

Lüdecke, D. (2017). Package 'esc'. https://cran.r-project.org/ 
Maldonado Moscoso, P. A., Castaldi, E., Burr, D. C., Arrighi, R., \& Anobile, G. (2020). Grouping strategies in number estimation extend the subitizing range. Scientific Reports, 10(1), 14979. https://doi.org/10. 1038/s41598-020-71871-5

Mandler, G., \& Shebo, B. J. (1982). Subitizing: An analysis of its component processes. Journal of Experimental Psychology: General, 111(1), 1-22.

McCandliss, B. D., Yun, C., Hannula, M., Hubbard, E. M., Vitale, J., \& Schwartz, D. (2010). Quick, how many? fluency in subitizing and "groupitizing" link to arithmetic skills, Poster Presented at the Biennial meeting of the American Educational Research Association, Denver, CO, USA.

Mead, L. A., Mayer, A. R., Bobholz, J. A., Woodley, S. J., Cunningham, J. M., Hammeke, T. A., \& Rao, S. M. (2002). Neural basis of the stroop interference task: Response competition or selective attention? Journal of the International Neuropsychological Society, 8(6), 735-742. https : // doi . org / 10 . 1017 / S1355617702860015

Morrissey, T. W., Hutchison, L., \& Winsler, A. (2013). Family income, school attendance, and academic achievement in elementary school. Developmental Psychology, 50(3), 741. https://doi.org/10.1037/ a0033848

Nieder, A., \& Miller, E. K. (2004). A parieto-frontal network for visual numerical information in the monkey. Proceedings of the National Academy of Sciences of the United States of America, 101(19), 7457-7462. https://doi.org/10.1073/pnas.0402239101

Nye, B., Konstantopoulos, S., \& Hedges, L. V. (2004). How large are teacher effects? Educational Evaluation and Policy Analysis, 26(3), 237-257. https://doi . org/10.3102/01623737026003237

Paliwal, V., \& Baroody, A. J. (2020). Cardinality principle understanding: The role of focusing on the subitizing ability. ZDM, 52(4), 649-661. https://doi.org/ 10.1007/s11858-020-01150-0

Siegler, R. S. (2007). Cognitive variability. Developmental Science, 10(1). https://doi.org/10.1111/j.14677687.2007.00571.x

Starkey, G. S., \& McCandliss, B. D. (2014). The emergence of "groupitizing" in children's numerical cognition. Journal of Experimental Child Psychology, 126, 120-137. https :// doi.org/10.1016/j.jecp. 2014. 03.006

Starkey, P., \& Cooper, R. G. (1995). The development of subitizing in young children. British Journal of Developmental Psychology, 13(4), 399-420. https:// doi.org/10.1111/j.2044-835X.1995.tb00688.x

Trick, L. M., \& Pylyshyn, Z. W. (1994). Why are small and large numbers enumerated differently? a limited- capacity preattentive stage in vision. Psychological Review, 101(1), 80-102. https://doi.org/10.1037/ 0033-295X.101.1.80

Vandierendonck, A. (2017). A comparison of methods to combine speed and accuracy measures of performance: A rejoinder on the binning procedure. $B e$ havior Research Methods, 49(2), 653-673. https : //doi.org/10.3758/s13428-016-0721-5

Vuokko, E., Niemivirta, M., \& Helenius, P. (2013). Cortical activation patterns during subitizing and counting. Brain Research, 1497, 40-52. https://doi .org/10. 1016/j.brainres.2012.12.019

Wagner, R. K., Torgesen, J. K., Rashotte, C. A., \& Pearson, N. A. (2010). Tosrec: Test of silent reading efficiency and comprehension. Pro-Ed.

Wege, T. E., Trezise, K., \& Inglis, M. (2021). Finding the subitizing in groupitizing: Evidence for parallel subitizing of dots and groups in grouped arrays. Psychonomic Bulletin $\mathcal{F}$ Review. https://doi.org/10. 3758/s13423-021-02015-7

Whitaker, K. J., Vendetti, M. S., Wendelken, C., \& Bunge, S. A. (2018). Neuroscientific insights into the development of analogical reasoning. Developmental Science, 21(2), e12531. https://doi.org/10.1111/ desc. 12531

Woltz, D. J., \& Was, C. A. (2006). Availability of related long-term memory during and after attention focus in working memory. Memory $\mathcal{E}$ Cognition, 34(3). https://doi.org/10.3758/BF03193587

Woodcock, R., McGrew, K., Schrank, F., \& Mather, N. (2007). Woodcock-johnson iii normative update. Rolling Meadows.

Younger, J., O’Laughlin, K. D., Anguera, J. A., Bunge, S. A., Ferrer, E., Hoeft, F., McCandliss, B. D., Mishra, J., Rosenberg-Lee, M., Gazzaley, A., \& Uncapher, M. R. (2021). Development of executive function in middle childhood: A large-scale, in-school, longitudinal investigation. PsyArXiv. https://doi.org/ 10.31234/osf.io/xf489 